\title{
Artifacts Removing From EEG Signals by Ica Algorithms
}

\author{
A. Srinivasulu ${ }^{1}$, M. Sreenath Reddy ${ }^{2}$ \\ ${ }^{1,2}$ (Electronics and Communication, MITS, Madanapalli/JNTUA, INDIA)
}

\begin{abstract}
Recent advances in computer hardware and signal processing have now a way to communicate with the outside world, but even with the last modern techniques, such systems still suffer communication Recent advances in computer hardware and signal processing have made possible the use of EEG signals or "brain waves" for communication between humans and computers The EEG is composed of electrical potentials arising from several sources. Each source (including separate neural clusters, blink artifact) projects a unique topography onto the scalp. These maps are mixed according to the principle of linear superposition. Here we attempt a Independent component analysis (ICA) of different algorithms to reverse the superposition by separating the EEG into mutually independent scalp maps, later removing their noise by set the threshold level and finally we classify the five mental tasks through the use of the electroencephalogram (EEG) by the neural network technique.
\end{abstract}

Keywords: EEG database, Independent component analysis (ICA) Algorithm, MAT LAB,

\section{Introduction}

Brain Computer Interface Technology: Present BCI's use EEG activity recorded at the scalp to control cursor movement, select letters or icons, or operate a neuroprosthesis. The central element in each BCI is a translation algorithm that converts electrophysiological input from the user into output that controls external devices. BCI operation depends on effective interaction between two adaptive controllers: the user who encodes his or her commands in the electrophysiological input provided to the BCI, and the computer which recognizes the command contained in the input and expresses them in the device control.

Current BCI's have maximum information transfer rates of 5-25 bits/min. Achievement of greater speed and accuracy depends on improvements in: Signal acquisition, Single trial analysis, Co-learning, Experimental paradigms for interpretable readable signals, Understanding algorithms and models within the context of the neurobiology.

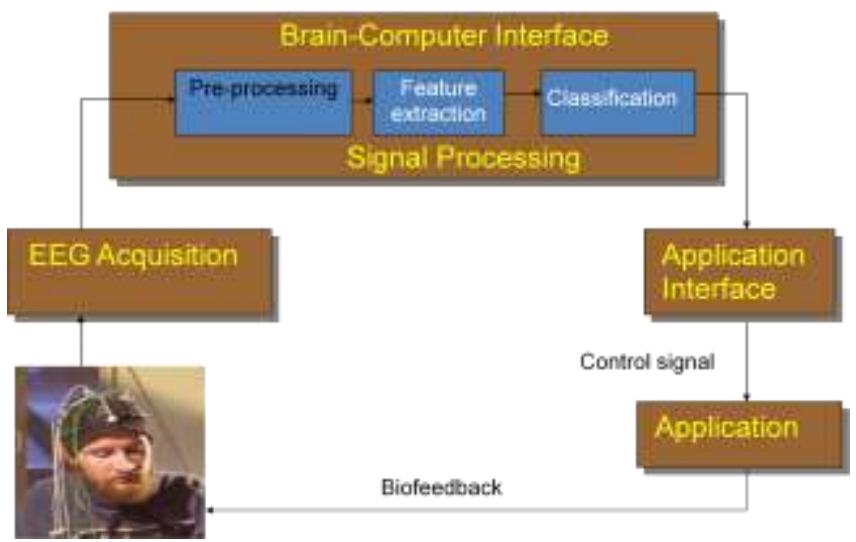

Fig. (1.1) Structure of Brain Computer Interface

The common structure of a Brain Computer Interface is shown in fig. (1) And the following are:

1) Signal Acquisition: the EEG signals are obtained from the brain through invasive or non-invasive methods (for example, electrodes). After, the signal is amplified and sampled.

2) Signal Pre-Processing: once the signals are acquired, it is necessary to clean them.

3) Signal Classification: once the signals are cleaned, they will be processed and classified to find out which kind of mental task the subject is performing

4) Computer Interaction: once the signals are classified, they will be used by an appropriate algorithm for the development of a certain application. 


\section{Implementation}

\subsection{EEG Signal Pre - Processing}

One of the main problems in the automated EEG analysis is the detection of the different kinds of interference waveforms (artifacts) added to the EEG signal during the recording sessions. These interference waveforms, the artifacts, are any recorded electrical potentials not originated in brain. There are four main sources of artifacts emission:

1. EEG equipment.

2. Electrical interference external to the subject and recording system.

3. The leads and the electrodes.

4. The subject her/himself: normal electrical activity from the heart, eye blinking, eyes movement, and muscles in general.

\subsection{Removing EEG artifacts by ICA algorithms}

Independent component analysis (ICA) is a relatively recent method for blind source separation (BSS), which has shown to outperform the classical principal component analysis (PCA) in many applications. In particular, it has been applied for the extraction of ocular artifacts from the EEG, where principal PCA could not separate eye artifacts from brain signals, especially when they have comparable amplitudes.

This method presents some advantages compared to other rejection methods, such as:

1. ICA separates EEG signals including artifacts into independent components based on the characteristics of the data, without relying on the availability of one or more "clean" reference channels for each type of artifact. This avoids the problem of mutual contamination between regressing and regressed channels.

2. ICA-based artifact removal can preserve all of the recorded trials, a crucial advantage over rejectionbased methods when limited data are available, or when blinks and muscle movements occur too frequently, as in some subject groups.

3. Unlike regression methods, ICA-based artifact removal can preserve data at all scalp channels, including frontal and particular sites.

Nevertheless, it is important to keep in mind that it also has some inherent

2.3 Limitations, Such As

1. ICA can decompose at most $\mathrm{N}$ sources from $\mathrm{N}$ scalp electrodes. Usually, the effective number of temporally-independent signals contributing to the scalp EEG is unknown, and it is likely that observed brain activity arises from more physically separable effective sources than the available number of EEG Electrodes.

2. The assumption of temporal independence used by ICA cannot be satisfied when the training data set is too small, or when separate topographically distinguishable Phenomena always occur concurrently in the data. In the latter case, simulations show that ICA may derive a component accounting for their joint occurrence, plus Separate components accounting for their periods of solo activation. Such confounds imply that converging behavioral or other evidence must be obtained before concluding that spatio-temporally overlapping ICA components measure Neuron-physiologically or functionally distinct activities.

3. ICA assumes that the physical sources of artifactual and neural activity contributing to EEG signals are spatially stationary through time. In general, there is no reason to believe that cerebral and artifactual sources in the spontaneous EEG necessarily remain stationary over time or occurrences.

4. The fact that this method needs more computations compared to a rejection approach, together with the inherently real-time nature of the EEG Brain Computer Interface makes its use a more difficult alternative.

\subsection{Algorithms for ICA}

There are a lot of kinds of algorithm for ICA. Some of them are follows:

-The algorithm relies on batch computations minimizing or maximizing contrast functions based on higherorder cumulate.

- The algorithm based on stochastic gradient methods, which may have implementations in neural networks.

\subsubsection{Fast-ICA}

The Fast-ICA [1] algorithm belongs to the family of fix-point algorithms for ICA, which is based on the iteration to search for the maximum of the non-Gaussianity of variables.

$\odot \quad$ Fixed-point algorithm

- Optimizes negentropy (negative entropy) or kurtosis to measure non-Gaussianity.

( ) Negentropy of $X$ is defined by

$\mathrm{J}(\mathrm{X})=\mathrm{H}\left(\mathrm{X}_{\text {gauss }}\right)-\mathrm{H}(\mathrm{X})$

○ Good statistical properties, but computationally difficult.

- Approximations of negentropy 
$J_{\mathrm{G}}(x)=\left(E\{G(x)\}-E\left\{G\left(x_{\text {gauss }}\right)\right\}\right)^{2}$ where $\mathrm{G}($.$) is a non quadratic function$

$$
G_{1}(u)=\frac{1}{a_{1}} \log \cosh a_{1} u \quad G_{2}(u)=-\exp \left(-\frac{u^{2}}{2}\right) \quad G_{3}(u)=\frac{1}{4} u^{4}
$$

The steps of Fast ICA algorithm

1. Centering the input observation signal $\mathrm{x}$

2. Whitening the centered signal $\mathrm{x}$

3. Initializing weight matrix $\mathrm{w}$, and set convergence error $\boldsymbol{\varepsilon}$

4. Update weight matrix $\mathrm{w}$. Using following iterative formula

$$
W_{i}^{+}=W_{i}-\mu\left[E\left\{x g\left(W_{i}^{T} x\right)\right\}-\beta W_{i}\right] /\left[E\left\{g^{\prime}\left(W_{i}^{T} x\right)\right\}-\beta\right]
$$

Where $\beta=E\left\{W_{i}^{T} x g\left(W_{i}^{T} x\right)\right\}$,

$$
g(z)=\frac{d G(z)}{d z}, \quad g^{\prime}(z)=\frac{d^{2} G(z)}{d z^{2}}
$$

5. Normalize weight vector w: $W_{i}^{+}=W_{i}^{+},\left\|W_{i}^{T}\right\|$

6. If $\left|W_{k+1}-W_{k}\right|>\varepsilon$ algorithm is not reach convergence, repeat steps (4) and (5);

7. Get the separation matrix $\mathrm{w}$

\subsubsection{JADE [2]}

Joint Approximate Diagonalization of Eigen matrices

$\rightarrow$ Built on cumulants - based contrast function.

Fourth order cross- cumulants for zero-mean random variables $x_{i}, x_{j}, x_{k}, x_{b}$, the cross-cumulants is defined as

$\operatorname{Cum}\left(x_{i}, x_{j}, x_{k}, x_{l}\right)=E\left[x_{i} x_{j} x_{k} x_{l}\right]-E\left[x_{i} x_{j}\right] E\left[x_{k} x_{1}\right]-E\left[x_{I} x_{k}\right] E\left[x_{j} x_{1}\right]-E\left[x_{I} x_{l}\right] E\left[x_{j} x_{k}\right]$

$\circ \quad$ Auto cumulants-Cum $\left(\mathrm{x}_{\mathrm{i}} \mathrm{x}_{\mathrm{i}} \mathrm{x}_{\mathrm{i}} \mathrm{x}_{\mathrm{i}}\right)=\mathrm{E}\left[\mathrm{x}_{\mathrm{i}} \mathrm{x}_{\mathrm{i}} \mathrm{x}_{\mathrm{i}} \mathrm{x}_{\mathrm{i}}\right]-\mathrm{E}\left[\mathrm{x}_{\mathrm{i}} \mathrm{x}_{\mathrm{i}}\right] \mathrm{E}\left[\mathrm{x}_{\mathrm{i}} \mathrm{x}_{\mathrm{i}}\right]-\mathrm{E}\left[\mathrm{x}_{\mathrm{i}} \mathrm{x}_{\mathrm{i}}\right] \mathrm{E}\left[\mathrm{x}_{\mathrm{i}} \mathrm{x}_{\mathrm{i}}\right]-\mathrm{E}\left[\mathrm{x}_{\mathrm{i}} \mathrm{x}_{\mathrm{i}}\right] \mathrm{E}\left[\mathrm{x}_{\mathrm{i}} \mathrm{x}_{\mathrm{i}}\right]$

$$
\begin{aligned}
& =\mathrm{E}\left[\mathrm{x}_{\mathrm{i}}^{4}\right]-3\left(\mathrm{E}\left[\mathrm{x}_{\mathrm{i}}{ }^{2}\right]\right)^{2} \\
\operatorname{Kurt}(\mathrm{X}) & =\mathrm{E}\left(\mathrm{X}^{4}\right)-3\left(\mathrm{E}\left(\mathrm{X}^{2}\right)\right)^{2}
\end{aligned}
$$

- $\operatorname{Kurt}(X)=0$ if $X$ Gaussian, $<0$ if sub and $>0$ if super

- jointly diagonalizing eigenmatrices of the kurtosis Steps in JADE

- Initialization. Estimate a whitening matrix $\mathrm{W}^{\wedge}$ and $\operatorname{set} \mathrm{Z}=\mathrm{W}^{\wedge} \mathrm{X}$.

- Form statistics. Estimate a maximal set $\quad\left\{\hat{Q}_{i}^{Z}\right\}$ of cumulants matrices.

- Optimize an orthogonal contrast. Find the rotation matrix $\hat{V}$ such that the cumulants matrices are as diagonal as possible, that is, solve

$$
\hat{V}=\arg \min \Sigma_{i} o f f\left(V^{T} \hat{Q}_{i}^{Z} V\right)
$$

- Separate. Estimate $\mathrm{A}$ as $\hat{A}=\hat{V} \hat{W}^{-1}$ and/or estimate the components as $\hat{S}=\hat{A}^{-1} X=\hat{V}^{T} Z$

\subsubsection{Infomax Algorithm [3]}

Principle

- Sources are assumed independent.

$\Rightarrow$ they don't have mutual information

- Therefore, minimizing the mutual information in observed signals will lead to the separated signals.

Mutual Information $>=0$ and zero if and only if the variables are statistically independent

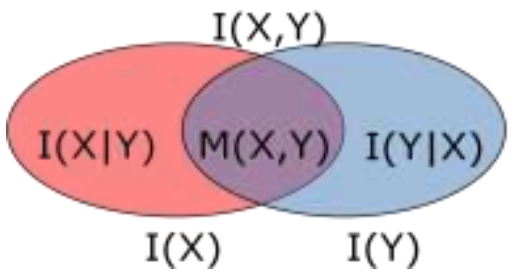

Information Measures

Information is given by, $I(X)=\int p(x) \log \frac{1}{p(x)} d x$

Entropy:

$$
\text { Joint Entropy: } \quad I(X, Y)=\int p(x, y) \log \frac{1}{p(x, y)} d x d y
$$


Mutual Information,

$$
M(X, Y)=I(X)+I(Y)-I(X, Y)
$$

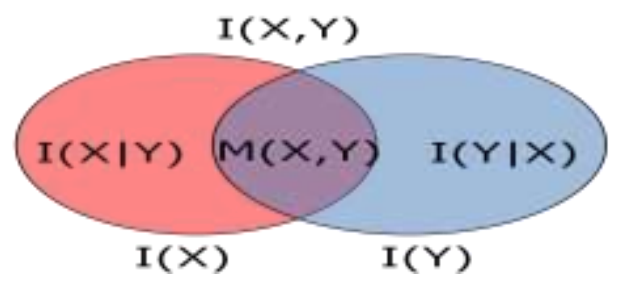

Minimizing Mutual Information $\approx$ Maximizing Joint Entropy/ Likelihood /Network Entropy

\subsubsection{Extended Infomax [2]}

$\circ \quad$ Extension of Infomax

- This preserves the ICA architecture of Infomax algorithm

$$
\Delta W=\left[1-K \tanh (u) u^{T}-u u^{T}\right] W \quad \begin{gathered}
\mathrm{K}=1: \text { Supergaussian } \\
\mathrm{K}=-1: \text { Subgaussian }
\end{gathered}
$$

\section{EEG Data Set: Mental Tasks}

$>\quad$ Resting task (Baseline)

$>\quad$ Imagined letter composing

$>\quad$ Mental multiplication

$>\quad$ Visualized counting

$>\quad$ Geometric object rotation

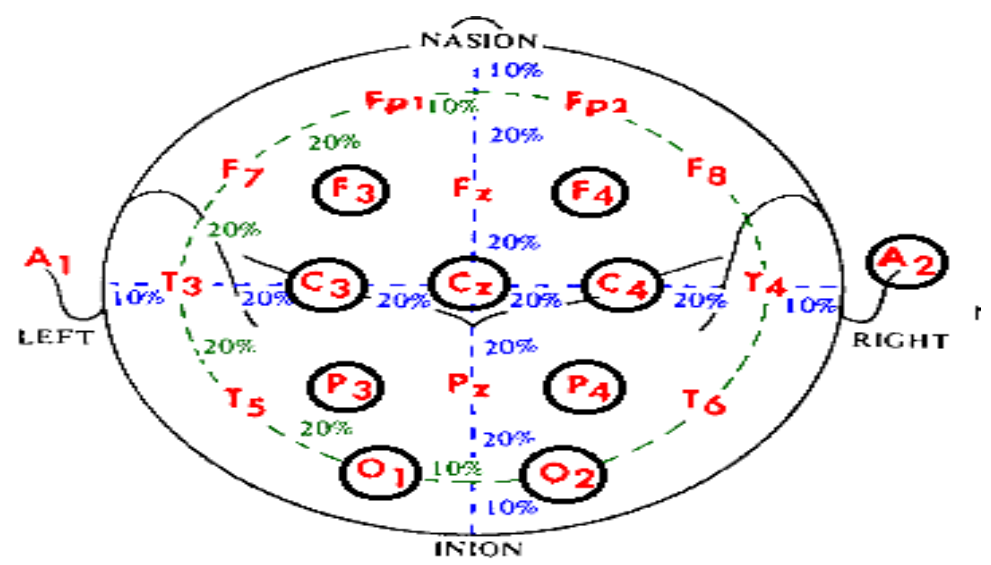

Fig.(2.1) Electrode placement on to the scalp Electrodes: C3, C4, P3, P4, O1, O2 and EOG

III. Results

Acquired Signals

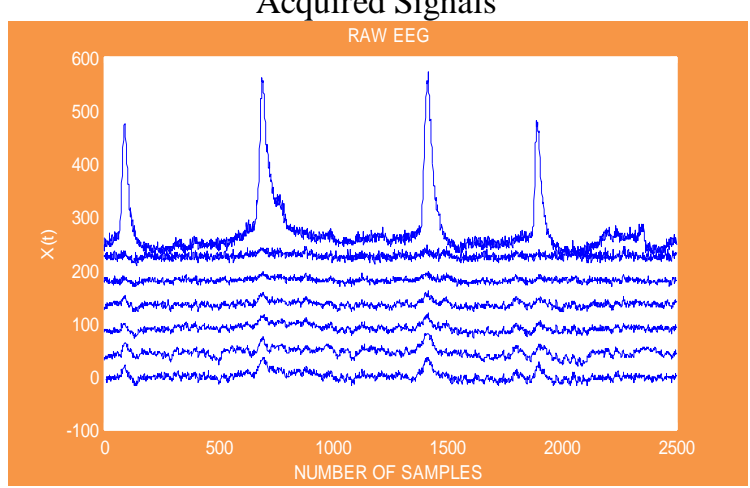

Fig. (3.1) Raw EEG signals 


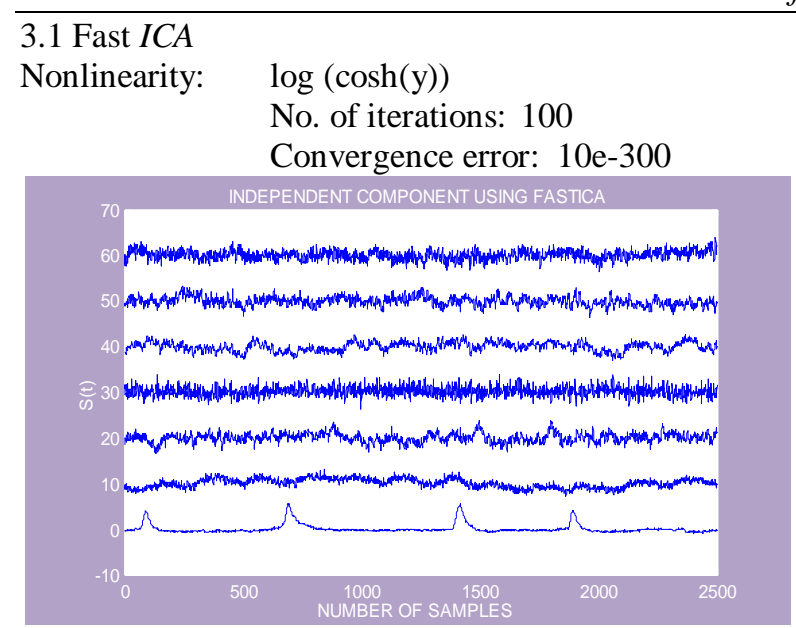

Fig.(3.2) Pure EEG signal by Fast ICA algorithm

\subsection{JADE}

No adjustable Parameters

\subsection{Infomax}

Convergence eeror $=1 \mathrm{e}-3$

Transformation function $=$ logistic sigmoid $=\frac{1}{1+e^{-u}}$

Number of iterations: 512

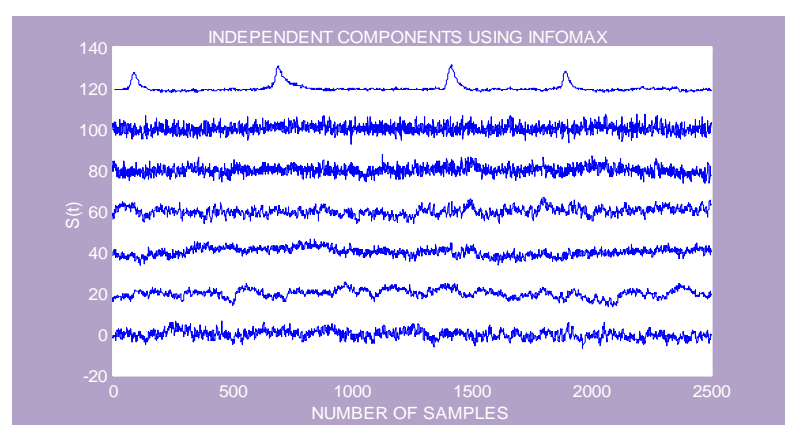

Fig.(3.4) Pure EEG signal by Infomax ICA algorithm
Fig.(3.3) Pure EEG s

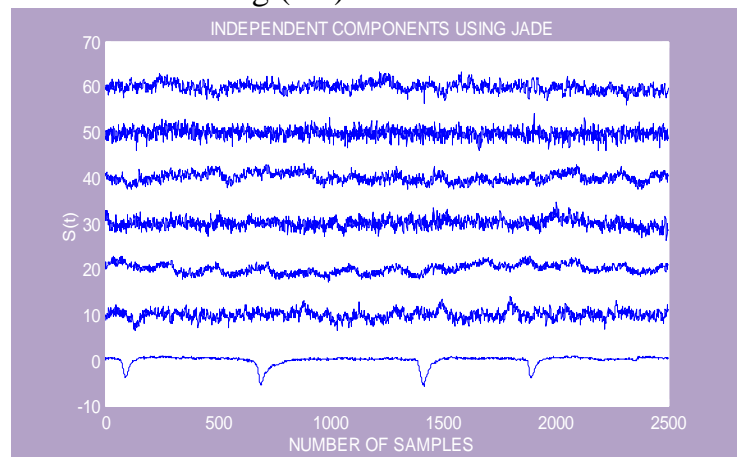

ignal by JADE ICA algorithm
3.4 Extended Infomax

Convergence error: $1 \mathrm{e}-3$

Number of iterations $=512$ Nonlinearity $=\tanh (\mathrm{u})$

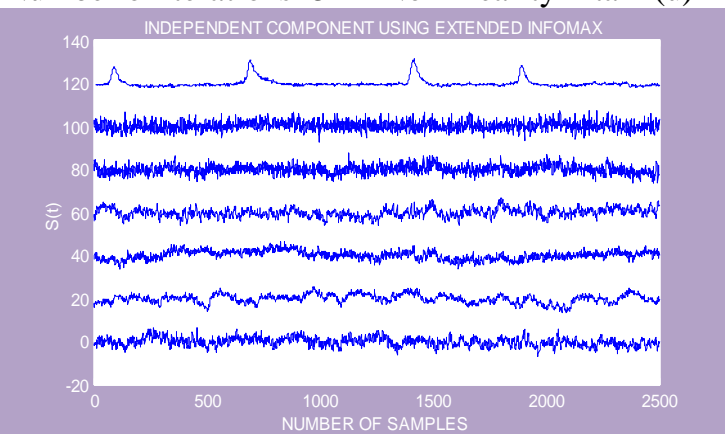

Fig. (3.5) Pure EEG signal by Extended Infomax ICA algorithm

\section{Reconstructed EEG}

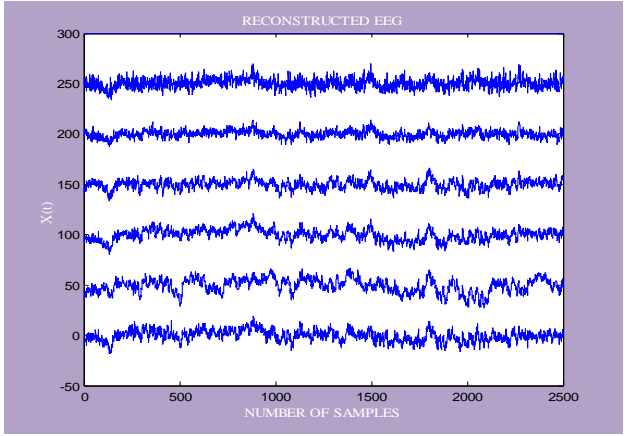

Fig(3.6):pure EEG signals without artifacts 
IV.

Conclusion

ICA is the central topic in this paper. EEG signals will maintain the similarity in their patterns when subject is performing the mental task. BCI systems using EEG as control signal suffers from the artifact problem. The traditional methods applied for remove artifacts can only compromise between eliminating artifacts and protecting useful signals so that the result is not very satisfying. However, ICA method can protect the useful signals as well as obviously weaken even entirely remove the artifacts in multi channel EEG signals, this characteristic of ICA is the key to get stable EEG patterns which can be used for mental task classification.

\section{Reference}

[1] Aapo Hyvarinen., "fast and Robust fixed point algorithm for independent component analysis," IEEE transactions on Neural Networks, Vol.10, pp.626-634, 1999.

[2] Amari , and Cichocki., "A new learning algorithm for blind source separation,” Advances in Neural Information Processing, MIT press, pp.757-763, vol.8, 1996.

[3] A.J.Bell and T.J.Sejnowski, "An information-maximization approach to blind separation and blind deconvolution," Neural Computation, vol.7, pp.1129-1159, 1995.

[4] T.W.Lee and T.J.Sejnowski, "Independent component analysis for sub-gaussian and super-gaussian mixtures," Proceedings of $4^{\text {th }}$ joint symposiums on Neural computations, vol.7, pp.132-139, 1997.

[5] Sarah M. Hosni, and Mahmoud E.gadallah., "Classification of EEG signals using different feature extraction techniques for mental-task BCI," Ain Shams university, Cairo, Egypt.

[6] Hyv $\square$ arinen A., Karhunen J., Oja E.: Independent Component Analysis. Wiley \{Interscience, 2001.

[7] M_ka S.: Numerick_e metody algebry. SNTL, 1985.

[8] Nev_s__malov_a S., __ Sonka K. a spol.: Poruchy sp_anku a bd_en_.. Maxdorf s.r.o., 1997 Data downloaded from: http://www.cs.colostate.edu/ anderson. 